\title{
ALGORITMO DE CLASSIFICAÇÃO DINÂMICA PARA O EVENTO DE PRISÃO DE COLUNA NA PERFURAÇÃO DE POÇOS OFFSHORE
}

\author{
Felippe Schuwartz Borba Marques \\ Universidade Federal Fluminense \\ fschuwartz@id.uff.br \\ Gilson Brito Alves Lima \\ Universidade Federal Fluminense \\ glima@id.uff.br \\ Fabiana Rodrigues Leta \\ Universidade Federal Fluminense \\ fabianaleta@id.uff.br \\ Claudio Benevenuto de C. Lima \\ PETROBRAS - Petróleo Brasileiro S.A. \\ clima@petrobras.com.br \\ Hardy Leonardo da Cunha Pereira Pinto \\ PETROBRAS - Petróleo Brasileiro S.A. \\ hardy@petrobras.com.br
}

\begin{abstract}
RESUMO
A perfuração de poços de petróleo Offshore em águas ultra profundas envolve diversas atividades pautadas em medidas de controle e segurança de poço, uma vez que eventos não desejados podem atrasar as operações e gerar tempos perdidos de operação. Dentre esses eventos críticos, a prisão de coluna é um acidente que coloca em risco as ferramentas da coluna, na tentativa de liberá-la, sendo necessário interromper a perfuração, para realizar procedimentos específicos de pescaria e liberação da mesma. A avaliação de tal evento crítico ainda hoje é feita pela sensibilidade e experiência do operador de campo, fato este que torna a identificação prévia de parâmetros críticos suscetíveis à falha. Neste sentido, torna-se interessante o estudo e desenvolvimento de ferramentas para avaliação dinâmica do evento com base em fatores quantitativos, reduzindo assim a subjetividade da análise. $\mathrm{O}$ presente trabalho apresenta uma proposta de algoritmo baseado no comportamento das principais variáveis relacionadas à prisão de coluna, onde é possível identificar condições favoráveis ou críticas à continuidade da perfuração. Desta forma, o estudo busca auxiliar a equipe de monitoramento da plataforma no suporte à decisão relacionada à identificação prévia do evento não desejado, a fim de proporcionar, a tempo, medidas de controle que evitem a prisão de coluna durante a perfuração.
\end{abstract}

Palavra-chave: Perfuração de Poços; Águas Ultra Profundas; Eventos Críticos; Avaliação Dinâmica, Prisão de Coluna. 


\begin{abstract}
The oil well drilling in Offshore and Ultra deep waters involves several activities based on measures control and well safety, since unwanted events can delay operations and generate non-productive operating times. Among these critical events, stuck pipe is a condition that puts the borehole tools in a risk. In an attempt to release it, for this operation is also necessary to stop drilling and perform specific procedures for fishing and releasing the tools. The drilling evaluation and the parameters control of such a critical event is still made today by the sensitivity and field operator experience, which makes the identification susceptible to failure. In this sense, it is interesting to study and develop tools for dynamic evaluation of critical events based on quantitative factors, thus reducing the subjectivity of the analysis. The present work presents an algorithm proposal based on the behavior of the main variables related to stuck pipe, where it is possible to identify favorable or critical conditions for the drilling continuity. In this way, the study seeks to assist the monitoring team of the platform in the support decision related to the previous identification of the undesired event, in order to provide in time, control measures while drilling to avoid the stuck pipe
\end{abstract}

Keywords: Well Drilling Offshore; Ultradeep Waters; Critical Events; Dynamic Evaluation; Stuck Pipe.

\title{
Como Citar:
}

MARQUES, F.S.B., LIMA, G.B.A., LETA, F.R., LIMA, C.B.C., PINTO, H.L.C.P. ALGORITMO DE CLASSIFICAÇÃO DINÂMICA PARA O EVENTO DE PRISÃO DE COLUNA NA PERFURAÇÃO DE POÇOS OFFSHORE. In: SIMPÓSIO DE PESQUISA OPERACIONAL E LOGÍSTICA DA MARINHA, 19., 2019, Rio de Janeiro, RJ. Anais [...]. Rio de Janeiro: Centro de Análises de Sistemas Navais, 2019. 


\section{INTRODUÇÃO}

As rochas reservatórios presentes na bacia de Santos, mas também na de Campos, que constituem o assim chamado Pré-Sal, possuem grande quantidade de óleo leve, conforme Jones e Chaves (2015), e ocupam uma área que se estende por 800 quilômetros do litoral brasileiro, sendo responsável pela agregação de reservas de 176 bilhões de barris de petróleo, volume significativamente maior que as atuais reservas nacionais, que, em 2017, foram de 12,415 bilhões de barris. Um dos fatores que contribuíram na exploração foi a redução no tempo de perfuração ao longo dos anos, em função do patamar assumido na competitividade dos projetos do Pré-Sal. Agregando a esse fato, a Petrobras, maior operadora brasileira de exploração de poços, divulgou em seu plano de negócios e gestão do quinquênio 2019-2023 a previsão de investir US\$ 84,1 bilhões, sendo 82\% dirigidos à área de exploração e produção (Lima e Lima, 2018).

Quando perfurando esse ambiente, operadores experimentam, com frequência, situações de tempo não produtivo (NPT) como prisão de coluna, perda de circulação, pescaria e desvios não desejados na trajetória do poço. O tempo perdido é dado pelo tempo que não acrescenta valor algum ao avanço da penetração, sendo que alguns especialistas o consideram também como tempo não produtivo.

Dentre esses eventos críticos, a prisão de coluna é um acidente que coloca em risco as ferramentas da coluna na tentativa de liberá-la, sendo necessário interromper a perfuração para realizar procedimentos específicos de pescaria e liberação da mesma. A avaliação de tal evento crítico ainda hoje é feita pela sensibilidade e experiência do operador de campo, fato este que torna a identificação prévia de parâmetros críticos suscetíveis à falha. Neste sentido, torna-se interessante o estudo e desenvolvimento de ferramentas para avaliação dinâmica do evento com base em fatores quantitativos, reduzindo assim a subjetividade da análise.

O presente trabalho tem como objetivo apresentar uma proposta de algoritmo baseado no comportamento das principais variáveis relacionadas à prisão de coluna, onde é possível identificar condições favoráveis ou críticas à continuidade da perfuração. Desta forma, o estudo busca auxiliar a equipe de monitoramento da plataforma no suporte à decisão relacionada à identificação prévia do evento não desejado, a fim de proporcionar a tempo medidas de controle durante a perfuração que evitem a prisão de coluna.

Para obter os resultados pretendidos neste estudo, foi adotada uma metodologia de processo para auxiliar desde a estruturação do problema até a obtenção dos resultados. A figura 1 apresenta as etapas que constituem o processo da pesquisa a partir do entendimento do problema até a proposta do algoritmo e discussão de resultados.

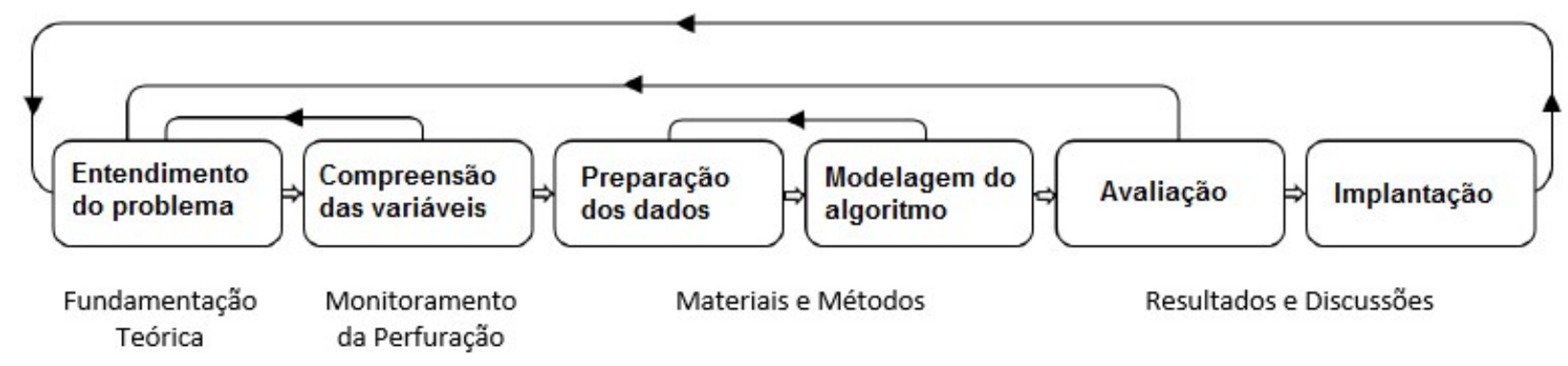

Figura 1 - Fluxo do desenvolvimento da pesquisa 


\section{FUNDAMENTAÇÃO TEÓRICA}

Esta sessão busca fundamentar as questões de prisão de coluna no processo de perfuração de poços, buscando apresentar uma compreensão dos conceitos ligados à perfuração, os diferentes modelos e dinâmicas do processo de prisão de coluna, bem como as formas e ferramentas utilizadas para medi-las, buscando identificar as especificidades para 0 caso da perfuração.

As pesquisas feitas sobre o tema principal deste estudo têm como objetivo identificar a literatura mais relevante sobre o problema da pesquisa e reunir as informações e dados que servirão de base para a construção da investigação proposta a partir do tema. Nesta pesquisa foi utilizada a base de dados do Portal de Periódicos da CAPES (Coordenação de Aperfeiçoamento de Pessoal de Nível Superior), bem como livros, revistas, jornais e sites. Em seguida um aprofundamento do tema foi realizado nas bases da OnePetro, OTC (Offshore Technology Conference) e SPE (Society of Petroleum Engineers) que possuem um foco maior na área de exploração, desenvolvimento e produção dos campos de petróleo.

A complexidade do tema envolve o entendimento e a correta interpretação dos dados recebidos para o estudo, desta forma, além da literatura, foi possível contar com a colaboração de especialistas em perfuração como engenheiros de perfuração e fiscais de sonda com sólida experiência de mercado. Embora a literatura auxilie no entendimento das variáveis relacionadas a prisão de coluna, o diagnóstico de coluna presa e os fatores precursores ao momento da prisão guardam certa subjetividade, que ainda nos dias de hoje, de forma qualitativa, são evidenciados pelos operadores da área de perfuração de poços. No sentido de realizar uma abordagem quantitativa, a proposta deste trabalho é propor um algoritmo que monitore e apresente em tempo real o risco de prisão de coluna durante a perfuração.

Ao longo do tempo, diversos autores buscaram entender o comportamento do poço durante a perfuração, dentre eles ressaltam-se alguns modelos de referência na literatura como o transporte dos sólidos, hidráulica e transferência de calor por Holmes e Swift (1970) e Raymond (1969), e análise do torque e arraste em poços direcionais por Johancsik; Friesen; Dawson (1984). Esses modelos são alimentados com dados em tempo real e simulam as condições do poço; a partir disso estima-se qual deve ser o valor esperado para o ECD, torque e arraste, peso sobre broca e pressão nas linhas de circulação. Atualmente, a análise dos problemas operacionais é feita por um especialista que busca interpretar tendências históricas e identificar comportamentos anormais que podem indicar situações críticas na operação (GHILARDI et al., 2013).

Na predição de eventos críticos durante a perfuração, a prisão de coluna é um dos que mais se destaca devido não somente ao tempo perdido, mas também a complexidade envolvida tanto no diagnóstico quanto nas técnicas para liberação das ferramentas presas ao fundo do poço. Os procedimentos necessários para tal medida, quando malsucedidos, podem em alguns casos inviabilizar a continuidade de exploração do poço, sendo necessário realizar cimentação e posterior abandono da locação.

\subsection{MONITORAMENTO DO PROCESSO DE PERFURAÇÃO}

Em determinados momentos os parâmetros ideais são comparados com os reais durante a perfuração. Quando os valores previstos pelos modelos estão de acordo com os reais entende-se que a operação acontece conforme o esperado; entretanto, se existe uma variação repentina não prevista no dado modelo, é possível concluir que algo de anormal está ocorrendo no poço, fato que pode representar uma ameaça a continuidade da perfuração. 
No monitoramento da perfuração, as principais variáveis abordadas na literatura associadas ao diagnóstico do risco de prisão de coluna estão descritas a seguir.

\subsubsection{Densidade Equivalente de Circulação (ECD)}

A densidade equivalente de circulação é uma boa referência para monitorar problemas de limpeza de poço e integridade das paredes da formação, pois a variação do ECD deve ser mínima, ou seja, registros de picos ou altas variações sem uma mudança no peso do fluido demonstram problema na capacidade de remoção de cascalhos (HESS, 2016).

No monitoramento do peso da lama é possível identificar quando a densidade do fluído está maior que o esperado através do aumento do ECD. Isto indica que a limpeza do poço não está conforme o programado devido a uma maior quantidade de cascalhos em suspensão, o que pode ter sido gerado por um dimensionamento ruim das propriedades dos fluído na fase de planejamento do poço.

\subsubsection{Torque e Arraste na Perfuração (T\&D)}

Os esforços gerados pela rotação da coluna e pelo peso aplicado sobre a broca, conforme figura 2, estão condicionados aos limites operacionais dos equipamentos envolvidos na perfuração. O planejamento prévio da operação de perfuração de um poço é fundamental para o sucesso da operação, normalmente estes parâmetros são comparados a poços de perfuração correlatos que obtiveram um bom desempenho quanto as medidas de torque e arraste da coluna.

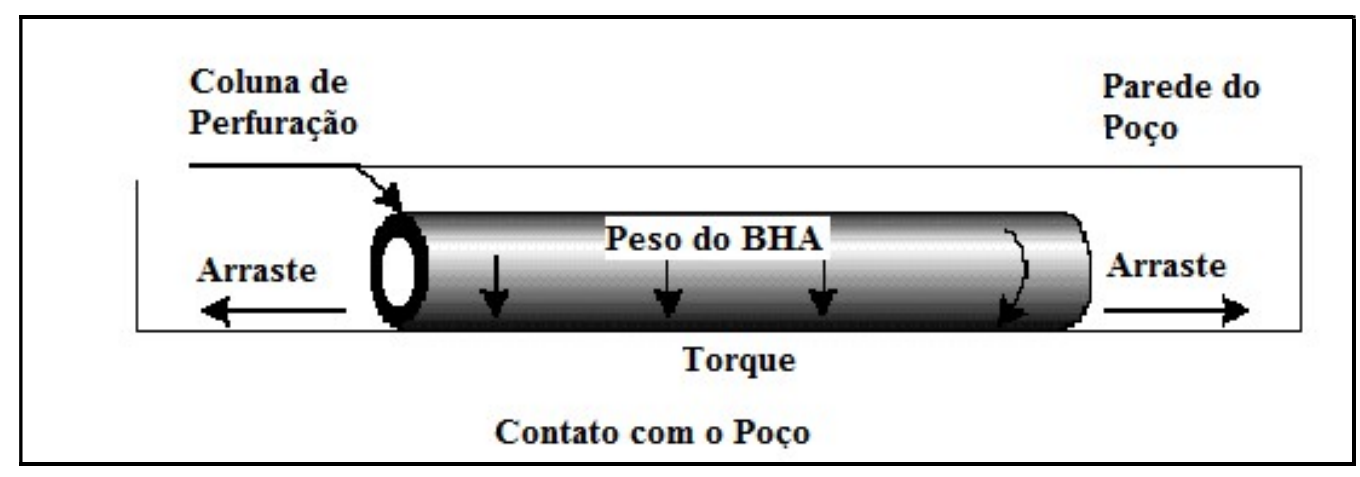

Figura 2 - Torque e arraste na Perfuração

A perda de energia durante a transferência do movimento rotacional da mesa de operação para a broca, ocasionada pela fricção, é a definição de torque, enquanto a diferença entre o peso da coluna parada e o peso da coluna em movimento é definida como arraste (AARRESTAD, 1994). Esta restrição no movimento está associada ao atrito entre os componentes da coluna de perfuração e o tipo da formação que forma a parede do poço. Equipamentos como estabilizadores ou alargadores são exemplos de elementos da coluna que podem gerar altos níveis de torque devido às suas grandes áreas de contato com a formação.

\subsubsection{Peso sobre broca}

O peso aplicado sobre a broca (WOB) tem relação direta com o desempenho da perfuração de poços. Se o peso aplicado na superfície não chega de maneira proporcional à broca, algo de inesperado pode estar restringindo essa transferência de peso (DUPRIEST et 
al., 2010). Dentre as diversas ferramentas que compõe a coluna rotativa, o Heavy Weight Drill Pipe e o Drill Collar (tubos com maior diâmetro externo e espessura) são responsáveis por adicionar o peso na coluna de perfuração que será transferido para a broca.

O peso na broca é normalmente associado com a carga da coluna liberada pelo operador na superfície sobre a broca, entretanto, a fricção causada pelo arraste reduzem o peso aplicado desde a superfície até a broca. Para contornar tal situação a indústria vem utilizando sensores próximos à broca para ter a certeza do peso exato aplicado sobre a mesma, e trabalhar assim em seus limites operacionais com o objetivo de obter a maior taxa de penetração

\subsubsection{Pressão nas linhas de Superfície (SPP)}

No processo de perfuração, a maioria dos eventos e problemas impactam na pressão das linhas de superfície segundo Ghilardi et al. (2013). Qualquer mudança nas condições da perfuração, como alteração nas propriedades do fluído, desmoronamento da formação, carregamento do espaço anular ou entupimento de jatos da broca refletem diretamente nos valores de pressão monitorados na plataforma.

Os sensores localizados nas linhas de superfície são responsáveis pela leitura da pressão gerada a partir das bombas de lama que deslocam o fluido desde a superfície pelo interior da coluna de perfuração, saindo pela broca, atingindo a formação e finalmente retornando pelo anular de volta a superfície. Neste momento qualquer obstrução no poço ou cascalhos acumulados de forma não desejada, ocasionará impactos na pressão das linhas ou será registrado pelo sensor e analisado pelos operadores durante a operação.

\subsubsection{Rotação na coluna de Perfuração (RPM)}

A quantidade de revoluções por minuto transmitidas à coluna de perfuração é determinada a partir de um estudo feito pelos especialistas para contribuir no bom desempenho da taxa de penetração e evitar problemas com suspensão de cascalhos. As recomendações dos valores permitidos para girar a coluna estão associados também aos limites operacionais de alguns equipamentos, como a broca de perfuração. Brocas de diamante, em geral, permitem um número de revoluções por minuto maior do que as brocas de cone, devido à sua estrutura robusta e dinâmica.

As revoluções aplicadas à broca são fundamentais no auxílio do corte da formação e na remoção dos cascalhos do anular para limpeza do poço; entretanto, elas são responsáveis também por induzir vibrações não desejadas. Segundo Mason e Chen (2007) em algumas faixas de rotação a coluna pode apresentar vibrações não desejadas como Stick Slip, que, se não mitigadas, podem contribuir com restrições ao giro da coluna.

\subsubsection{Vibrações do tipo Stick Slip (SS)}

O Stick Slip é a forma mais severa de vibração ocasionada pela torção da coluna (FEAR; ABBASSIAN, 1994), pois, após o vencimento da rotação ao torque que estava restringindo o giro, é percebida uma rotação superior àquela aplicada na superfície pelo operador, o que pode oferecer riscos aos limites operacionais antes estabelecidos e contribuir para a prisão de coluna. Outros tipos de vibrações também são conhecidos na indústria por causar danos às ferramentas e representar comportamentos indesejados na coluna de perfuração. Os mais conhecidos são: StickSlip, Lateral, Axial, Whirl. 


\section{MATERIAIS E MÉTODOS}

O estudo foi desenvolvido em um projeto de pesquisa na Universidade Federal Fluminense em parceria com a Petrobras para a predição de eventos de prisão de coluna, utilizando técnicas de inteligência artificial. Parte deste projeto teve como base o estudo das variáveis mais influentes no evento crítico estudado e, a partir do entendimento do comportamento das mesmas em momentos distintos, é possível identificar um comportamento padrão que antecede o fenômeno que deve ser evitado.

As etapas de compreensão, preparação, modelagem e avaliação dos dados foram executadas através do desenvolvimento de uma solução de software, utilizando a linguagem de programação Python ${ }^{\circledR}$, na versão 3.6. O Python ${ }^{\circledR}$ é uma linguagem de uso geral, de código aberto, e com funcionalidades adequadas para a criação de códigos e automatização de regras para a mineração de dados, principalmente com o uso de pacotes de computação científica (SciPy / NumPy), de manipulação de dados (Pandas) e geração de gráficos (Matplotlib), utilizados para os desenvolvimentos desta solução. O código gerado para aplicação do algoritmo proposto para análise do risco envolvido na prisão de coluna pode ser brevemente observado na figura 3.

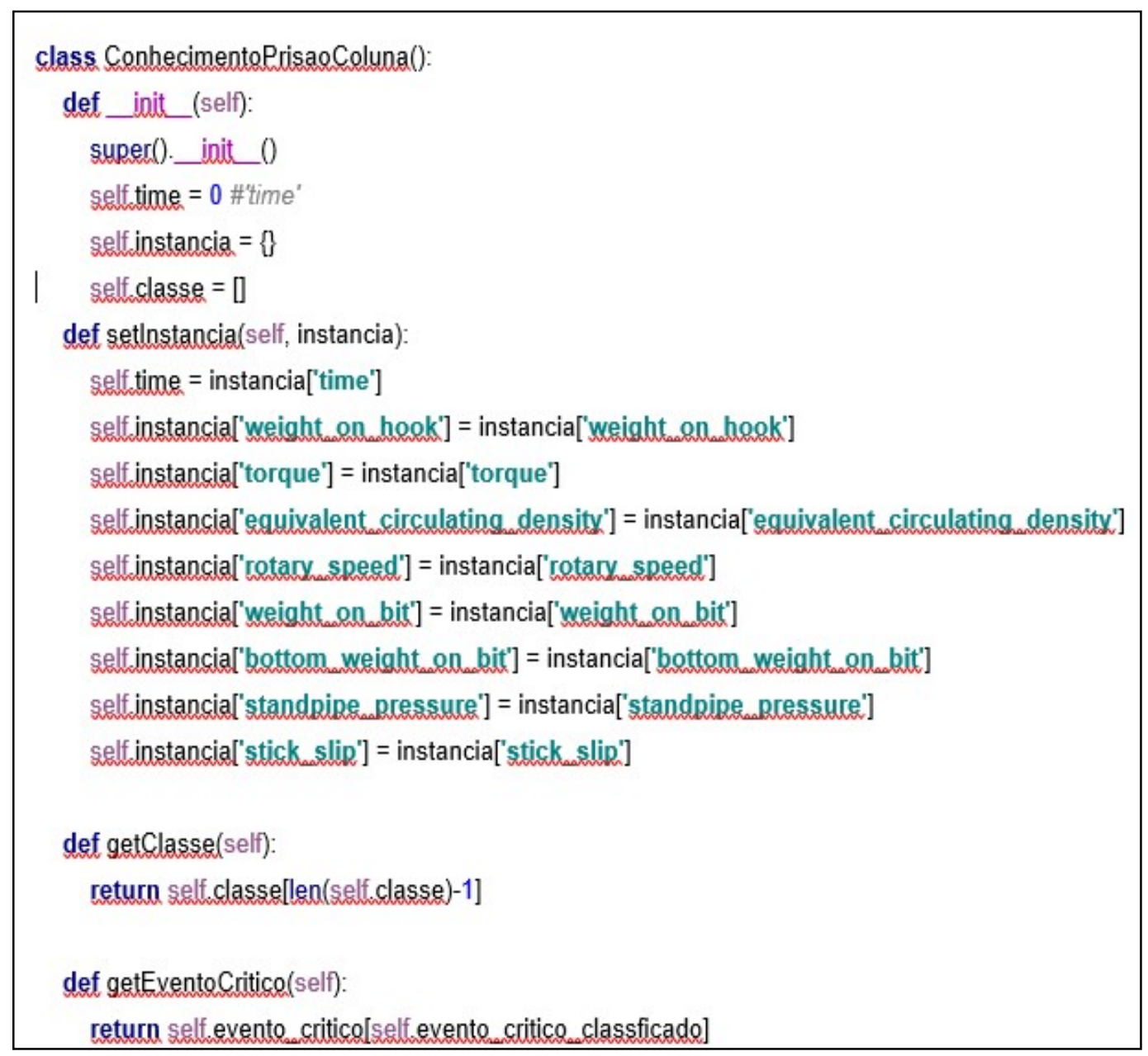

Figura 3 - Linguagem do algoritmo proposto

Para efetuar as manipulações nas etapas de compreensão, preparação, modelagem e avaliação dos dados, foi utilizada a linguagem de programação citada, além de alguns pacotes predefinidos de tratamento estatístico. Por questões de confiabilidade, apenas uma parte do código é apresentada, onde não estão concentrados os códigos de programação. 
Importante ressaltar que não foi o foco da pesquisa o uso da tecnologia. Desta forma, não serão detalhados ou explicados tecnicamente os comandos e funcionalidades da linguagem de programação utilizada no tratamento de dados e desenvolvimento da solução proposta para o evento estudado.

A partir dos dados provenientes dos sensores de campo é possível perceber o intervalo em que a perfuração está entrando em um momento crítico, passando por dificuldades ou restrições em seu avanço. $\mathrm{Na}$ plotagem dos gráficos conforme figura 4 e figura 5, relacionados aos valores enviados pelos sensores de algumas das variáveis observadas, é nítido perceber a evolução prejudicial ao movimento livre da coluna. Destacado em verde temos os valores normais e esperados, enquanto o circulado em amarelo já demonstra uma situação de risco, e finalmente o vermelho antecede o momento exato da prisão de coluna.

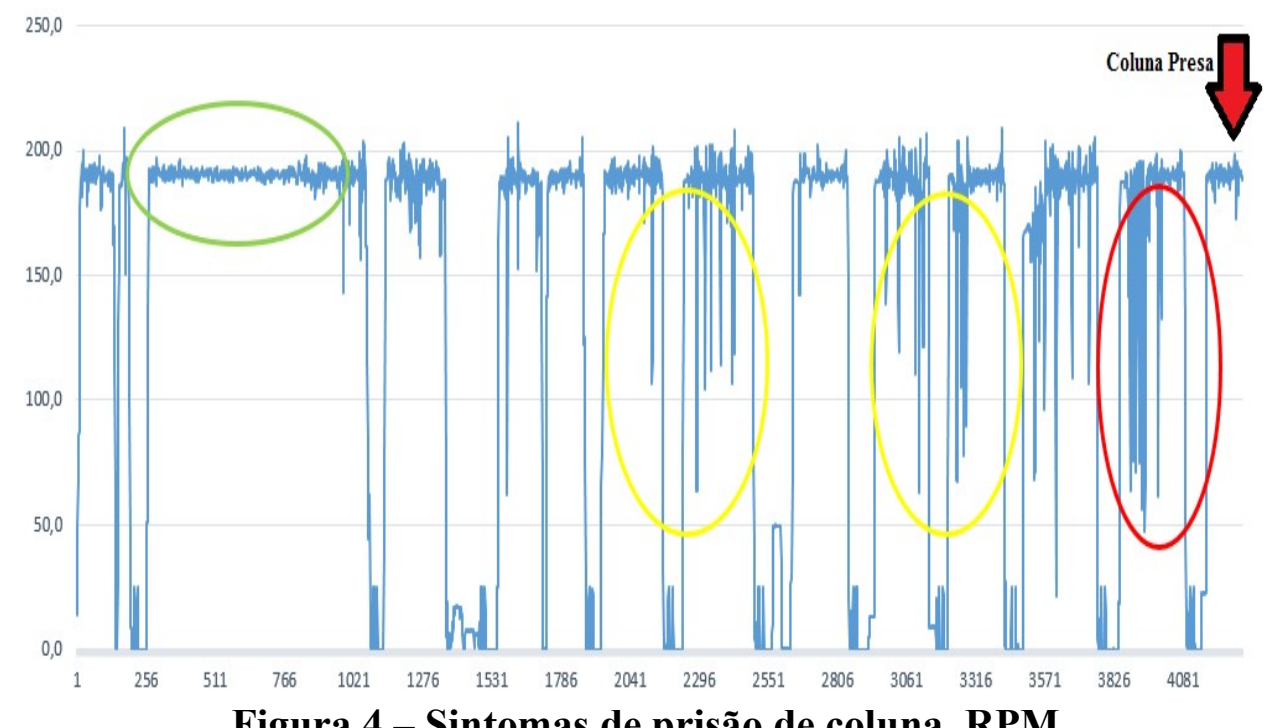

Figura 4 - Sintomas de prisão de coluna_RPM

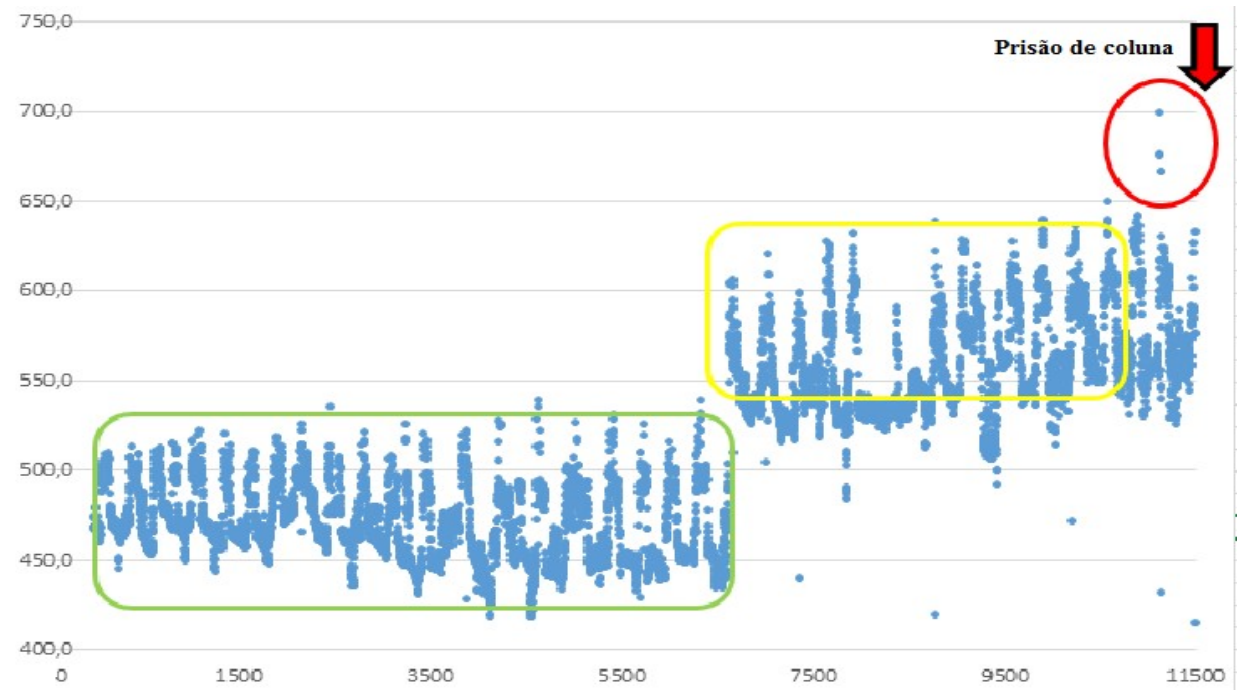

Figura 5 - Sintomas de prisão de coluna_Arraste

As informações de monitoramento servem de auxílio ao operador de campo para entender o comportamento do poço; no entanto, quando essas variáveis são observadas de forma isolada, não respaldam uma atitude imediata de interferir ou parar a perfuração, neste sentido foram observadas as seguintes oscilações dos valores das variáveis críticas pré- 
identificadas antes do evento crítico de prisão de coluna ocorrer, tratados abaixo como letras para não comprometer a confidencialidade dos dados referente a empresa estudada:

○ Maior Arraste da Coluna $\rightarrow\left[C_{1}, C_{10}\right]$ valores de Drag.

○ Aumento do Torque $\rightarrow\left[T_{1}, T_{10}\right]$ valores de Torque.

○ Variação de Densidade $\rightarrow\left[D_{1}, D_{10}\right]$ valores de ECD.

○ Restrição a Rotação $\rightarrow\left[R_{1}, R_{10}\right]$ valores de RPM.

○ Diferença de Peso $\rightarrow\left[P_{1}, P_{10}\right]$ valores de WOB.

- Aumento de Pressão Peso $\rightarrow\left[P r_{1}, P r_{10}\right]$ valores de SPP.

○ Nível de Vibração $\rightarrow\left[V_{1}, V_{10}\right]$ valores de StickSlip.

A proposta de algoritmo utiliza em sua métrica uma pontuação de valores relacionados a comportamentos dentro e fora do padrão normal de perfuração das variáveis observadas, ou seja, a partir da média da variável das últimas leituras dos sensores, os valores considerados dentro da zona crítica em relação às condições normais são pontuados com o valor mais alto associado a variável estudada. Desta forma foram coletados valores considerados críticos pelos especialistas, e proposta uma condição mínima necessária para que o valor lido pelo sensor seja considerado crítico em cada uma das variáveis selecionadas conforme Tabela 1.

Tabela 1: Medidas de Variação Crítica

\begin{tabular}{cccccccc}
\hline Poços $x$ & & & & & & \\
Variáveis & DWOB & Torque & RPM & ECD & Drag & SPP & StickSlip \\
\hline Poço 01 & 45,2 & 55,4 & 65,2 & 0,8 & 6,2 & 6,1 & 6 \\
Poço 02 & 70,6 & 55,2 & 80,1 & 1,4 & 5,5 & 4,3 & 5 \\
Poço 03 & 50,4 & 60,5 & 70,6 & 1,2 & 5,1 & 7,2 & 6 \\
Poço 04 & 55,6 & 45,1 & 65,4 & 0,7 & 6,7 & 5,1 & 6 \\
Poço 05 & 60,3 & 50,3 & 75,2 & 1,2 & 4,3 & 3,2 & 6 \\
Poço 06 & 40,2 & 55,4 & 55,2 & 1 & 6,4 & 5,3 & 5 \\
Poço 07 & 50,5 & 45,1 & 60,5 & 0,8 & 7,5 & 7,3 & 6 \\
Poço 08 & 55,2 & 60,2 & 80,2 & 0,95 & 5,2 & 4,2 & 6 \\
Poço 09 & 45,6 & 45,6 & 60,3 & 0,7 & 4,3 & 6,5 & 6 \\
Poço 10 & 40,3 & 50,7 & 80,5 & 1,2 & 4,4 & 5,1 & 5 \\
\hline Variação & \multicolumn{7}{c}{} \\
Média (\%) & 50,45 & 52,95 & 68 & 0,975 & 5,35 & 5,2 & 6 \\
\hline Variação (\%) & $\geq X_{1} \%$ & $\geq X_{2} \%$ & $\geq X_{3} \%$ & $\leq X_{4} \%$ & $\geq X_{5} \%$ & $\geq X_{6} \%$ & $\geq X_{7}$ \\
Crítica &
\end{tabular}

O algoritmo tem por objetivo pontuar o valor maior quando a última leitura satisfaz as condições críticas da variação média do comportamento crítico, para isso é estabelecida uma regra de comportamento baseado nas observações feitas durante a perfuração, ou seja, se o registro mais recente do sensor for maior do que a variação média dos últimos valores, este valor deve ser considerado fora das condições normais de perfuração. Nos gráficos de monitoramento é possível perceber que os momentos circulados em amarelo e vermelho são os intervalos críticos que contam mais pontos para o grau de risco associado à prisão de coluna.

\subsection{ATRIBUIÇÃo DE IMPORTÂNCIA ÀS VARIÁVEIS}


Para analisarmos a significância e hierarquia das variáveis relacionadas como fundamentais no evento de prisão de coluna é utilizado o método de análise hierárquica, o AHP (Analytic Hierarchy Process): decomposição e síntese das relações entre os critérios até que se chegue a uma priorização de seus indicadores, aproximando-se de uma melhor resposta de medição (Saaty, 1991).

Segundo Grandzol (2005) o método agrega valor em um projeto, ao tratar prioridades, de parâmetros e seleção de alternativas. O método é fundamentado na habilidade humana de fazer julgamentos sobre problemas diversos. A ideia principal da teoria da análise hierárquica introduzida por Saaty é a sintetização do estudo de sistemas a uma sequência de comparação aos pares. A relevância do método realiza-se no processo de tomada de decisões, minimizando suas falhas

Segundo Saaty (1994), a vantagem do método é que os valores provenientes das comparações paritárias são baseados na experiência profissional dos especialistas, intuição ou até mesmo dados físicos, desta forma, o AHP pode lidar com aspectos qualitativos e quantitativos de um mesmo problema de decisão. A partir da estrutura hierárquica montada na tabela 2 os especialistas fazem as comparações paritárias atribuindo um valor de importância a cada variável relacionada a outra.

Esta atribuição de valor busca agregar peso a todas variáveis em conjunto, tornando a análise mais robusta e não apenas relacionada a um único critério. No final da leitura os demais sensores selecionados irão colaborar para associar um grau de risco ao cenário monitorado. Para isso foram consultados especialistas do setor de perfuração que respaldaram a comparação paritária entre as variáveis mais influentes, com o objetivo de atribuir um peso a cada variável no algoritmo proposto.

Tabela 2: Comparação Paritária de Variáveis

\begin{tabular}{|c|c|c|c|c|c|c|c|c|c|c|}
\hline $\begin{array}{c}\text { Prisão de } \\
\text { Coluna }\end{array}$ & Torque & DRAG & RPM & Arraste & ECD & SPP & SS & Prioridade & $\begin{array}{c}\text { Critério } x \\
\text { Prioridade }\end{array}$ & $\lambda$ \\
\hline Torque & 1 & 1 & 1 & 2 & 2 & 3 & 3 & 0,212 & 1,494 & 1,004 \\
\hline DRAG & 1 & 1 & 1 & 2 & 2 & 3 & 3 & 0,212 & 1,494 & 1,004 \\
\hline RPM & 1 & 1 & 1 & 2 & 2 & 3 & 3 & 0,212 & 1,494 & 1,004 \\
\hline Arraste & $1 / 2$ & $1 / 2$ & $1 / 2$ & 1 & 1 & 2 & 2 & 0,116 & 0,812 & 1,003 \\
\hline ECD & $1 / 2$ & $1 / 2$ & $1 / 2$ & 1 & 1 & 2 & 2 & 0,116 & 0,812 & 1,003 \\
\hline SPP & $1 / 3$ & $1 / 3$ & $1 / 3$ & $1 / 2$ & $1 / 2$ & 1 & 1 & 0,066 & 0,459 & 1,001 \\
\hline SS & $1 / 3$ & $1 / 3$ & $1 / 3$ & $1 / 2$ & $1 / 2$ & 1 & 1 & 0,066 & 0,459 & 1,001 \\
\hline
\end{tabular}

Os valores obtidos para a média aritmética da coluna do Vetor de Consistência ( $\lambda$ máx), o Índice de Consistência (CI), o Índice Randômico (RI) e a Relação de Consistência (CR), estão registrados na Tabela 3, finalizando, o teste de consistência:

Tabela 3: Teste de Consistência

\begin{tabular}{|c|c|c|c|}
\hline$\lambda \operatorname{Max}$ & $\mathrm{n}$ & $\mathrm{Cl}$ & $\mathrm{CR}$ \\
\hline 7,02 & 7 & 0,003 & $0,25 \%$ \\
\hline
\end{tabular}

Através do teste de consistência realizado pôde-se constatar que a comparação pareada do decisor obteve uma Relação de Consistência de $0,25 \%$, que atende ao requisito de Saaty (1994), de que uma inconsistência aceitável deve ser menor ou igual a 0,1.

No final da leitura os demais sensores selecionados irão colaborar para associar um grau de risco ao cenário monitorado. Para isso foram consultados especialistas do setor de 
perfuração que respaldaram a comparação paritária entre as variáveis mais influentes, com o objetivo de atribuir um peso a cada variável do algoritmo proposto.

A pontuação individual de cada variável tem por objetivo pontuar o valor maior quando a última leitura satisfaz as condições críticas da variação média do comportamento, para isso é estabelecida uma regra individual para cada variável em questão, descrita a seguir como parte do algoritmo proposto, baseado nas observações feitas durante a perfuração.

O algoritmo possui uma variação em sua pontuação que vai de 7 até 21 , guardada as devidas proporções relacionadas a importância das variáveis, que representam uma escala associada ao grau de risco em uma eventual prisão de coluna. Quanto mais próximo de 7, menor a chance da coluna ficar presa; em contrapartida, quanto mais perto da pontuação de 21, maior a possibilidade de prisão de coluna, conforme indicação na tabela 4 .

Tabela 4 - Pontuação do risco associado pelo Algoritmo

\begin{tabular}{rrrrrrrrrrrrrrrr}
\hline 7 & 8 & 9 & 10 & 11 & 12 & 13 & 14 & 15 & 16 & 17 & 18 & 19 & 20 & 21 \\
\hline \multicolumn{3}{c|}{ Baixo Risco } & \multicolumn{3}{c}{ Médio Risco } & & \multicolumn{5}{c}{ Alto Risco } \\
\hline
\end{tabular}

O monitoramento dos dados permite que, em média a cada 10 segundos, sejam enviados dados das ferramentas de MWD, bem como dos sensores de Mudlogging dispostos na superfície. A partir desta frequência e do algoritmo proposto a seguir é possível manter um acompanhamento em tempo real das condições referentes ao risco de uma prisão de coluna ocorrer. A figura 5 apresenta um extrato do algoritmo de classificação desenvolvido para o processo de prisão mecânica de coluna.

SE Peso no Gancho dentro das condições de variação crítica $\geq X_{1}$ ENTÃO grau de risco <- grau de risco $+Y_{1}$ CASO CONTRÁRIO grau de risco $<-$ grau de risco $+Z_{1}$

SE Torque dentro das condições de variação crítica $\geq X_{2}$ ENTÃO grau de risco <- grau de risco $+Y_{2}$ CASO CONTRÁRIO grau de risco <- grau de risco $+Z_{2}$

SE Rotação na Coluna dentro das condições de variação crítica $\leq X_{3}$ ENTÃO grau de risco <- grau de risco $+Y_{3}$ CASO CONTRÁRIO grau de risco $<-$ grau de risco $+Z_{3}$

Figura 5 - Sintomas de prisão de coluna

\section{RESULTADOS E DISCUSSÕES}

O algoritmo foi aplicado para 8 poços, 4 com prisão de coluna e 4 sem o evento estudado. Percebe-se que antes do momento crítico, as variáveis possuem uma tendência de elevar a pontuação do algoritmo até 16 pontos, valor este que representa uma perfuração crítica em pelo menos 3 variáveis. Enquanto nos poços sem prisão de coluna a reta permanece sem uma angulação acentuada, demonstrando que não houve aumento de risco relacionado ao acidente. A seguir são apresentados a aplicação do algoritmo para poços com prisão de coluna, na figura 6 .

Fica claro a partir da linha de tendência observada nos gráficos que no início da perfuração as variáveis críticas possuem um somatório em pontuação menos expressiva, em quantidade e frequência. A partir de um dado momento, em cada um dos poços é possível perceber o somatório da pontuação do algoritmo em valores mais altos, o que faz levantar a 
linha de tendência e indicar que o fenômeno crítico da prisão de coluna ganha força e oferece risco elevado à perfuração.

Após a pontuação do algoritmo dinâmico nos poços com e sem prisão de coluna é possível perceber a importância da associação do grau de risco a inclinação da linha de tendência, uma vez que essa representa o risco tendo como referência pontuações anteriores. A representação das linhas de tendência ao longo do cenário da perfuração permite que o operador possa conjugar a pontuação momentânea com a evolução do risco relacionado a prisão de coluna ao longo da perfuração e ter uma noção do aumento ou não da criticidade relacionada a prisão de coluna

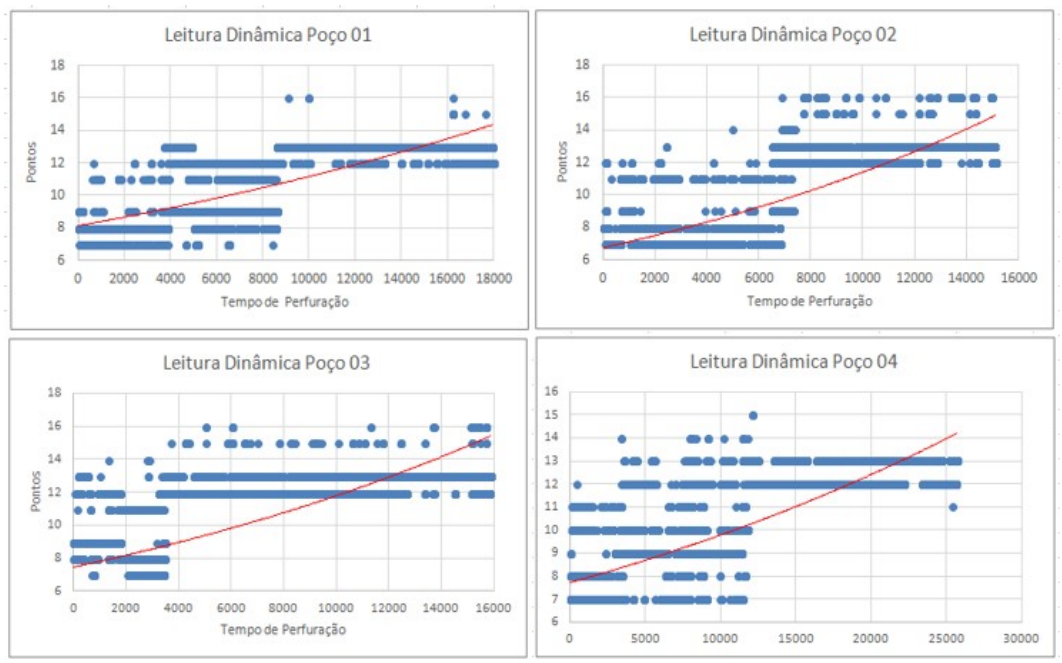

Figura 6 - Algoritmo em poços com prisão de coluna

O algoritmo foi também aplicado para 4 poços que não tiveram a coluna presa, desta forma é possível observar a diferença na pontuação ocorrida e na construção da linha de tendência a partir dos pontos marcados pelo monitoramento e aplicação do algoritmo utilizado. O comportamento dos demais poços estão representados pelos exemplos das 4 seguintes perfurações na figura 7 .

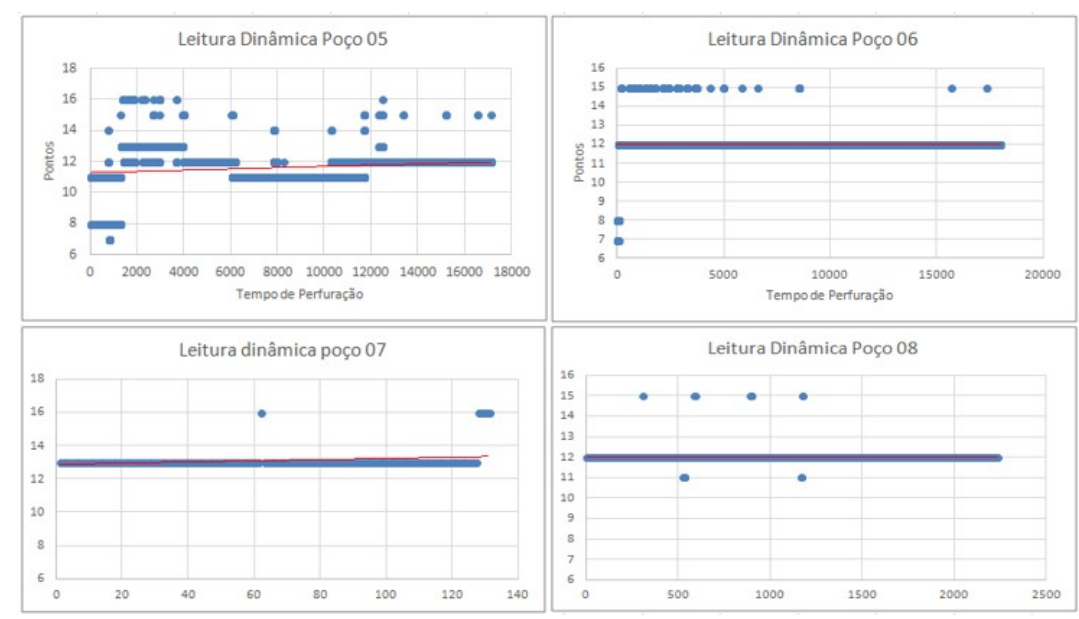

Figura 7 - Algoritmo em poços sem prisão de coluna

Pode ser observado que o comportamento da linha de tendência para um poço que não possui prisão de coluna é totalmente diferente daquele com prisão. À medida que a poço avança, não é notado o aumento da criticidade da perfuração. É interessante perceber que existem pontuações altas na faixa dos 16 pontos, entretanto, elas não são constantes e não 
aumentam sua incidência com o passar do tempo.

Nos poços em que não ocorre a prisão é possível perceber que a pontuação começa com valores mais altos, ou seja uma ou mais das variáveis monitoradas já apresentam um valor acima da média considerada normal para aquela variável, esse fato evidencia que a perfuração já apresenta uma condição crítica associada, que pode ser justificado por um dimensionamento inadequado da coluna de perfuração, gerando torque, arraste, vibrações ou ainda propriedades do fluído incapazes de realizar a limpeza do poço, entretanto, percebe-se que as condições do poço não evoluem para uma pontuação ainda maior, o que permite que a coluna não fique presa e a perfuração siga até a profundidade desejada.

\section{CONCLUSÃO}

Este trabalho objetivou delinear uma proposta para avaliação dinâmica do evento crítico de prisão de coluna. Através do entendimento do comportamento crítico das variáveis selecionadas foi possível observar através da pontuação do algoritmo proposto o comportamento diferenciado na perfuração entre os poços com e sem prisão de coluna. Para observar tal comportamento o algoritmo foi aplicado aos poços e uma linha de tendência foi traçada para ambos os casos.

Os poços sem prisão de coluna, apesar de possuírem algumas pontuações mais altas, apresentam em sua tendência uma reta de baixa inclinação, que é explicada por uma condição de poço constante, ou seja, as variáveis críticas monitoradas para que o evento ocorra permaneceram sem alterações significativas durante todo o intervalo de perfuração da fase de 17.5 polegadas em que o poço foi monitorado.

Já os poços que tiveram a coluna presa demonstram que no início da operação as condições de poço são brandas, entretanto, com o avanço da perfuração, essas condições mudam e as variáveis críticas passam a levar a linha de tendência para um patamar mais elevado. A partir da não intervenção do operador, leva ao acúmulo de fatores críticos durante a operação que resulta em condições não favoráveis ao avanço da coluna, consecutivamente presa ao poço.

A pontuação do algoritmo e a linha de tendência têm por objetivo proporcionar ao operador de campo uma maior ciência sobre a possibilidade do evento da prisão de coluna ocorrer durante a perfuração. Nesse sentido, com a observação da tendência crítica da reta, é possível que medidas de prevenção sejam tomadas a tempo de evitar o evento indesejado e contribuir para a redução de custos que poderão advir.

Para futuros trabalhos, recomenda-se a identificação de outros eventos indesejados durante a perfuração, assim como a prisão diferencial, a perda de circulação ou falha de ferramentas de fundo de poço, que podem possuir indicativos e evidências que permitam ser monitorados por variáveis que explicam ou contribuem para esses eventos; e, a partir de uma avaliação dinâmica pautada em um algoritmo, fazer o monitoramento do evento a fim de evitá-lo e contribuir na redução de custos na perfuração.

\section{REFERÊNCIA BIBLIOGRÁFICA}

\section{[1] AARRESTAD, T. V. Torque and drag-two factors in extended-reach drilling.} Journal of Petroleum Technology, v. 46, n. 09, p. 800-803, 1994.

[2] CRESWELL, J. W. Projeto de pesquisa: métodos qualitativo, quantitativo e misto. Trad. Luciana de Oliveira Rocha. 2. ed. Porto Alegre: Artmed, 2007Terceira referência. 
[3] DUPRIEST, F. E.; ELKS, W. C.; OTTESEN, S.; PASTUSEK, P. E.; ZOOK, J. R.; APHALE, C. R. Borehole-Quality Design and Practices To Maximize Drill-Rate Performance. SPE Annual Technical Conference and Exhibition, p. 18, 2010.

[4] FEAR, M. J.; ABBASSIAN, F. Experience in the Detection and Suppression of Torsional Vibration From Mud Logging Data. European Petroleum Conference, p. 433-448, 1994. Disponível em: http://www.onepetro.org/doi/10.2118/28908-MS.

[5] GHILARDI, P.; DE MARI, A.; LINS, D. G.; MARTINS, A. L.; GANDELMAN, R. A.; ARAGAO, A. F. L.; TEIXEIRA, G. T.; CASTANEDA, R. M.; LENZ, C. Real Time Drilling Data Diagnosis Implemented In Deepwater Wells - A Reality. Offshore Technology Conference, OTC 24275, p. 1-9, 2013.

[6] GHILARDI, P.; DE MARI, A.; LINS, D. G.; MARTINS, A. L.; GANDELMAN, R. A.; ARAGAO, A. F. L.; TEIXEIRA, G. T.; CASTANEDA, R. M.; LENZ, C. Real Time Drilling Data Diagnosis Implemented In Deepwater Wells - A Reality. Offshore Technology Conference, OTC 24275, p. 1-9, 2013.

[7] GRANDZOL, John R. Improving the Faculty Selection Process in Higher Education: A Case for the Analytic Hierarchy Process. IR Applications. Volume 6. Association for Institutional Research (NJ1), 2005.

[8] HESS, J. Pipe Sticking Prediction Using LWD Real-Time Measurements. IADC/SPE Drilling Conference and Exhibition, n. March, p. 1-3, 2016. Disponível em: http://www.onepetro.org/doi/10.2118/178828-MS.

[9] HOLMES, C. S.; SWIFT, S. C. Calculation of circulating mud temperatures. Journal of Petroleum Technology, v. 22, n. 06, p. 670-674, 1970.

[10] JOHANCSIK, C. A.; FRIESEN, D. B.; DAWSON, R. Torque and drag in directional wells-prediction and measurement. Journal of Petroleum Technology, $v$. 36, n. 06, p. 987-992, 1984.

[11] JONES, C. M.; CHAVES, H. A. F. Assessment of yet-to-find-oil in the Pre-Salt area of Brazil. In: 14th International Congress of the Brazilian Geophysical Society, Anais...2015.Primeira referência. Primeira referência. Primeira referência. Primeira referência. Primeira referência.

[12] LIMA, P. C. R.; LIMA, P. G. da C. Alternativas para o setor petrolífero e para a Petrobras. 2018. Disponível em: < http://www2.camara.leg.br/atividadelegislativa/estudos-e-notas-tecnicas/publicacoes-da-consultoria-legislativa/areas-daconle/tema16/alternativas-para-o-setor-petrolifero-e-para-a-petrobras $>$.Segunda referência.

[13] MASON, C. J.; CHEN, D. C.-K. Step Changes Needed To Modernize T\&D Software. SPE/IADC Drilling Conference, v. 2, n. SPE/IADC 104609, p. 1-11, 2007.

[14] RAYMOND, L. R. Temperature distribution in a circulating drilling fluid. Journal of Petroleum Technology, v. 21, n. 03, p. 333-341, 1969.

[15] SAATY, Thomas Lorie; VARGAS, Luis Gonzalez. Prediction, projection, and forecasting: applications of the analytic hierarchy process in economics, finance, politics, games, and sports. Kluwer Academic Pub, 1991. 
